\title{
El avance tecnológico y su impacto en la educación inicial.
}

\section{Technological advance and its impact on early education.}

\author{
Efraín Velasteguí López. ${ }^{1}$
}

\section{Resumen.}

La educación inicial y sus avances tecnológicos en el ecuador ha desempeñado gran potencial en los niños con un $23.9 \%$ en la educación inicial, $12,3 \%$ en los adultos y esos no dejas con un 4,8\% en la educación superior. Estos datos nos reflejan como los avances tecnológicos han venido revolucionando a nivel académico.

Por ese motivo la tarea docente implica identificar las capacidades que los nativos digitales necesitan para ser eficaces en esta nueva cultura o modo de ser; recreando metodologías y materiales basados en entornos digitales, aprovechando al máximo su eficacia, pero conservando nuestra humanidad.

La educación inicial tiene un conjunto de prácticas pedagógicas innovadoras que se han ido consolidando a lo largo del tiempo. En tal sentido, la integración de las nuevas tecnologías a los procesos educativos con los niños pequeños constituye un reto y una oportunidad para modelar y optimizar nuestra práctica docente, respondiendo a la adaptación más rápida e importante que ha experimentado el cerebro en miles de años de evolución.

Es importante analizar el carácter que las personas y, como tales, también los maestros y los niños establecen con las tecnologías. Cada vez que las usamos para modificar un estado de cosas, a su vez somos modificados por ellas. Por tanto, a jugar con nuevas tecnologías, también se aprende.

Palabras claves: Impacto, avances tecnológicos, educación inicial.

\section{Abstract.}

Initial education and its technological advances in Ecuador have played great potential in children with $23.9 \%$ in early education, $12.3 \%$ in adults and those do not leave with $4.8 \%$

\footnotetext{
${ }^{1}$ Ciencia digital, Ambato, Ecuador, luisefrainvelastegui@cienciadigital.org
} 
in higher education. These data reflect how technological advances have been revolutionizing at the academic level.

For this reason, the teaching task involves identifying the capabilities that digital natives need to be effective in this new culture or way of being; recreating methodologies and materials based on digital environments, making the most of their effectiveness, while preserving our humanity.

Initial education has a set of innovative pedagogical practices that have been consolidated over time. In this sense, the integration of new technologies to educational processes with young children is a challenge and an opportunity to model and optimize our teaching practice, responding to the most rapid and important adaptation that the brain has experienced in thousands of years. Evolution.

It is important to analyze the character that the people and, as such, also the teachers and the children establish with the technologies. Every time we use them to modify a state of things, in turn we are modified by them. Therefore, to play with new technologies, you also learn.

Keywords: Impact, technological advances, initial education.

\section{Introducción}

\section{La educación}

No podríamos desarrollar este trabajo sin dejar claro el concepto de educación que no es más que un proceso permanente e integral de interacción mediante el cual hay un aprendizaje individual y/o grupal. También se define como un proceso formativo, que prepara para asumir conocimientos y orienta hacia una visión crítica y transformadora, pero en realidad es el acto en que los sujetos aprenden a aprender; se refiere a la creciente autonomía de las personas" a adquirir conocimientos.

Según: Desevilla A. 2017., Martinez J. 2016., Reyes M. 2016., Rowan C. 2018., Rodriguez I. 2017\& Sigdel S. 2017 Afirman: La tecnología forma parte ya de nuestra cultura y algunos niños se encontrarán por primera vez con el texto escrito a través de la televisión, el ordenador o los teléfonos móviles, artefactos que forman ya parte de la cultura popular en nuestro país. Aunque existen en el mercado una gran variedad de productos destinados a niños de preescolar que incorporan aspectos tecnológicos, como teclados musicales, grabadoras, juguetes programables y controlados por radio, controles remotos, teléfonos, televisiones, el objetivo de este texto es centrarse en la utilización del ordenador. A partir de este reconocimiento se propone a los agentes educativos generar "espacios educativos significativos", entendidos como ambientes de aprendizaje estructurados, retadores y generadores de múltiples experiencias que favorecen en niños y niñas la construcción de nuevos conocimientos.

"La tecnología en la educación cada día avanza más y más, que para los niños hoy en día el manejo de la electrónica se le hace sencilla manejarla, cada institución en la educación inicial han desarrollado el sistemas de enseñanza avanzada gracias a los avances tecnológicos en la educación inicial. Los niños desarrollan competencias como la 
creatividad gracias al acceso que tienen a diferentes herramientas tecnológicas, con el fin de proponer metas deseadas en la educación infantil."

La revolución digital y tecnológica está transformando todos los sectores de la sociedad, incluido el de la educación. Estamos inmersos en lo que ya se denomina Cuarta Revolución Industrial, una transformación para la que, más temprano que tarde, habrá que adaptarse y formarse para poder ejercer profesiones que, en la actualidad, ni siquiera existen. Para afrontar esta nueva realidad digital, es imprescindible transformar el modelo educativo y asentarlo sobre unos cimientos basados en la innovación y la tecnología.

Grafico 1: Educación inicial y sus avances tecnológicos.

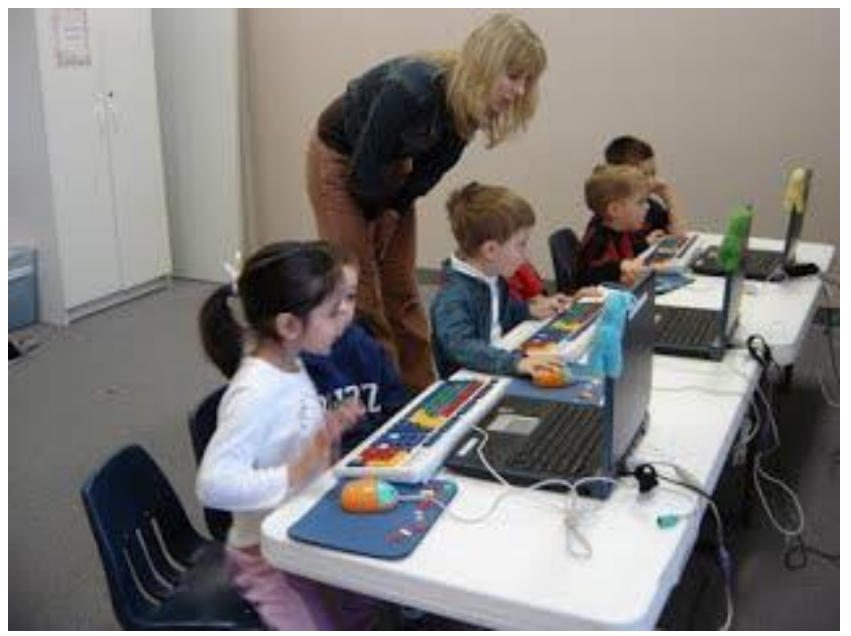

Autor: Natalie Liñayo

Fuente: https://sites.google.com/a/correo.unimet.edu.ve/2-equipo-4-eac-14152fgtce04//rsrc/1425952866280/home/impacto-de-las-tic-en-el-sistema-deeducacion-preescolar-eneuu/TIc $\% 20 y \% 20$ preescolar.jpg?height $=239 \&$ width $=320$

Hoy las familias son diferentes. La influencia de la tecnología en una familia del siglo XXI está fracturando su base y causando una desintegración de los valores fundamentales que hace tiempo eran el tejido que las unía. Los padres tienen que hacer juegos malabares con la escuela, el trabajo, la casa y la vida social, y dependen enormemente de las tecnologías de la comunicación, la información y el transporte para lograr unas vidas más rápidas y eficientes. Las tecnologías del entretenimiento (televisión, internet, videojuegos, iPads, teléfonos móviles) han avanzado con tal rapidez que las familias

apenas se han dado cuenta del enorme efecto y los grandes cambios que han supuesto en su estructura familiar y su estilo de vida.

Mientras más temprano se inician los niños en el uso de tecnologías digitales, menos se desarrollan habilidades sociales tales como la capacidad de leer la mente del otro a través de la mirada, la lectura de claves no verbales, la pragmática (adecuar con rapidez la conducta al contexto), la empatía y la atención espacial (recoger velozmente datos del contexto. En la actualidad la forma de enseñar se ha ido complementando con ayuda de los medios tecnológicos que están al alcance de las instituciones educativas, además puede observarse un interés innato por parte de los niños para utilizar estos medios. 
Grafico 2: Influencia de la tecnología en los niños

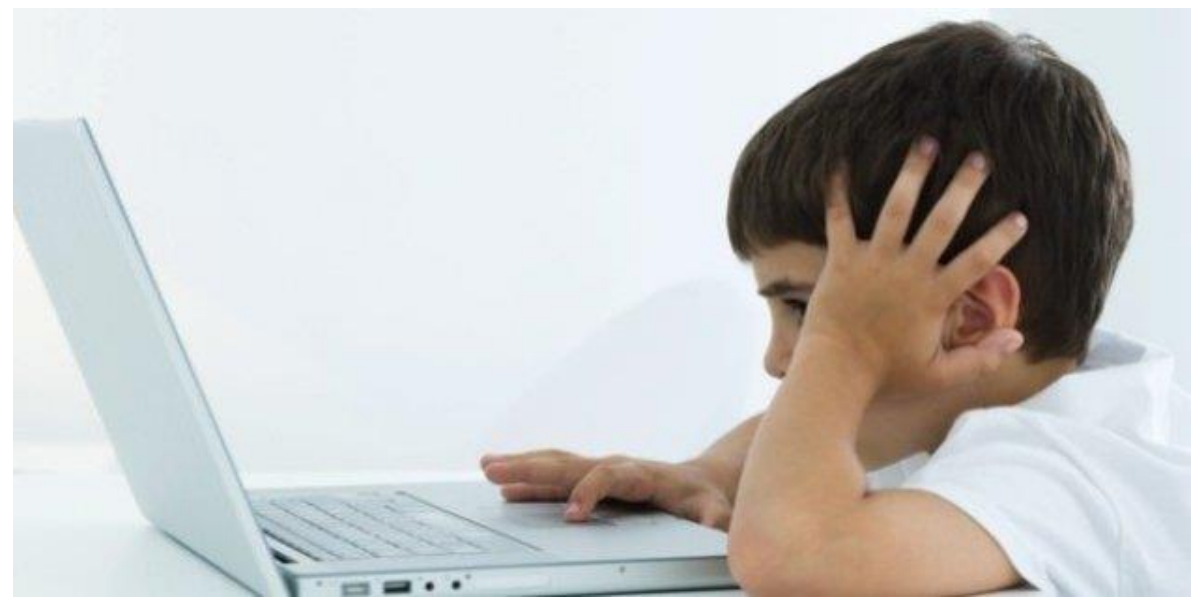

Autor: Rowan, 2018

Fuente: https://www.huffingtonpost.es/cris-rowan/influencia-de-la-tecnologianinos_b_4043967.html

"Los niños en edad escolar son los que más utiliza y disfrutan las nuevas tecnologías, favorecen los de los dispositivos preferidos como teléfono celulares reproductores ya que son fácil de usarlo."

Grafico 3: Actualidad de la tecnología en la educación.

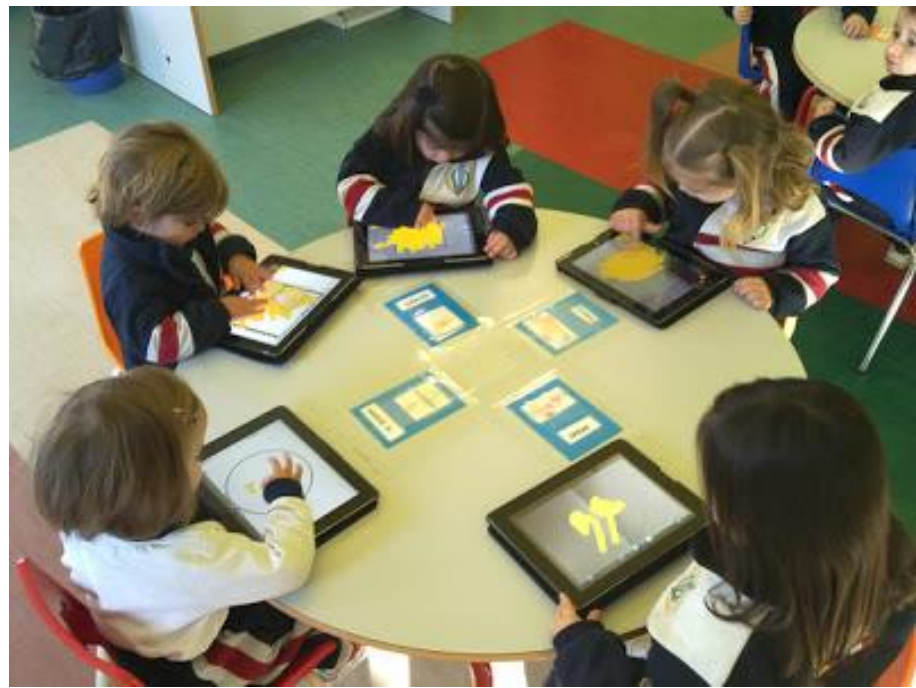

Autor: Monserrat García

Fuente: http://3.bp.blogspot.com/-

ZcTWCKrXKq8/UEIZF9iVfTI/AAAAAAAAAMA/6Vu7L0NtExY/s400/ipad+ en+la+edu.jpg

Según Garcia M. 2013., Fernandez R. 2015., Huidribo J. 2018., Jarvis E. 2017., Muntz A. 2017 \& Rosa A. 2016 afirman: Hoy en día, la tecnología está en todas partes y durante los próximos años la inteligencia artificial continuara desarrollándose, creando unos sistemas de reclutamiento más transparentes, eficientes e inteligentes. También seguirán y desaparecen otros que quedaras obsoleto, junto con la demografía y la 
globalización, la tecnología es una de las tres tendencias que redefine los mercados de trabajo.

"En los últimos años, la evolución se ha visto deslumbrado por la aparición de las tecnologías de la información la actividad tecnológica se ha convertido en la mayor influencia de las personas en todo el mundo gracias a los avances tecnológicos que ha impactado."

Definimos tecnología como el conjunto de procedimientos y recursos de gran complejidad y sofisticación que caracterizan a la técnica desde el siglo XVIII la diferencia fundamental respeto a la técnica es la aplicación de las teorías de los avances tecnológicos. La tecnología ha invadido todos los ámbitos de nuestra existencia. Esto ha puesto que la sociedad en la que vivimos se caracterice por un rasgo fundamental. Dominada por una omnipresencia.

Grafico 4: Tendencia Tecnológica

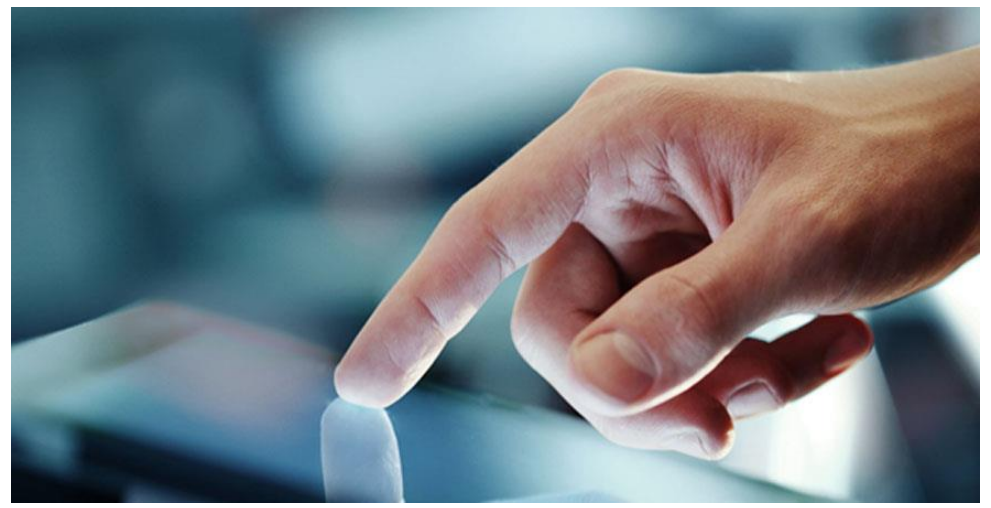

Autor: (Muntz, 2017)

Fuente: https://www.randstad.es/tendencias360/el-impacto-de-la-tecnologia-esmayor-de-lo-que-imaginas/

Actualmente, la tecnología ha penetrado en todas las sociedades, formando parte consustancial de ellas y, básicamente, uno de los mayores cambios que ha introducido es el de facilitar la comunicación interpersonal, con dispositivos y entre ellos, y un acceso rápido e instantáneo a la información, se esté donde esté.

La llegada de la tecnología a la vida de los seres humanos genero múltiples cambios: las relaciones sociales. En la era de la inmediatez y la información, la tecnología se convirtió en protagonista de diferentes experiencias de los seres humanos. Está presente en las relaciones sociales, en la educación y en las jornadas laborales.

Grafico 4: Tendencia Tecnológica en la educación inicial. 


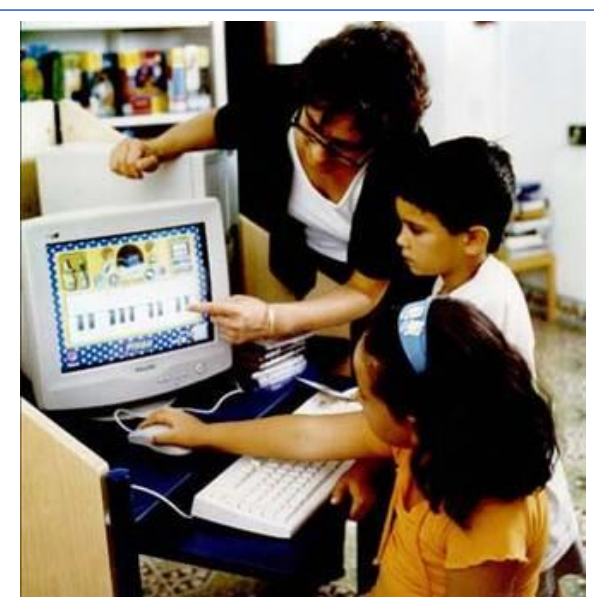

Autor: (Garcia, 2013)

Fuente: $\underline{\text { http://1.bp.blogspot.com/- }}$ WWK9LzTXzmY/USVxBbYq44I/AAAAAAAAAA0/fOTPk2qdj8/s1600/7 g.jpg/

Las practicas tradicionalistas en el nivel preescolar van quedando atrás, ya que el modelo educativo que actualmente se maneja por disposición oficial educativa tiene un carácter flexible, por lo que para favorecer las competencias en los niños, los docentes pueden idear y crear situaciones didácticas innovadoras en las modalidades de implementación de proyectos, talleres, etc., en donde cada vez más se observa el uso de tecnologías de uso habitual por los niños.

Grafico 5: Avances Tecnológica en la educación inicial.

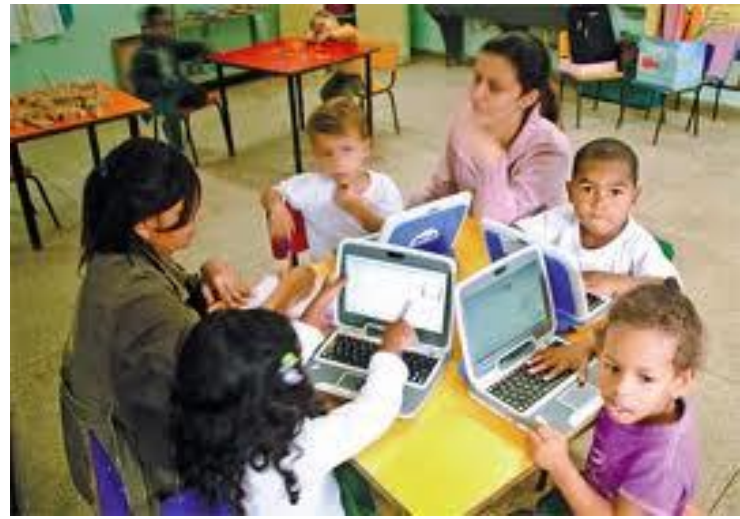

Autor: Educación inicial

Fuente:http://2.bp.blogspot.com/-HfCX3_ErX0/UR2gyAM_Q3I/AAAAAAAAABE/96RcIaY1Sz8/s1600/\%C3\%ADndice .jpg.

Gracias al alto crecimiento tecnológico en las últimas décadas en la sociedad, tras una larga trayectoria de innovaciones y adaptaciones tecnológicas, la tecnología se ha convertido en objetos de interés para el diseño en las instituciones educativas.

La observación del entorno que nos rodea ha sido una constante en la condición humana $\mathrm{y}$, como resultado que ha permitido estructurar un amplio campo de conocimiento en el mundo cultural de las sociedades en función del avance tecnológico. 
"En el mundo que vivimos hoy, las tecnologías en la educación inicial se han convertido en unos de los mayores factores más relevantes en cuanto el uso institucional educativas y muchas personas que puede hacer uso de la tecnología solo piensa en intente mas no en sus herramientas y ventajas tecnológicas que sirven para el manejo de la información, también la tecnología es el conjunto de conocimientos técnicos para un mayor aprendizaje en la vida estudiantil, empezando desde el inicio con las avances tecnológico en la educación inicia así fortalecer sus conocimiento gracias a las avances tecnológico en la educación.

Tabla 1: De datos estadísticos de educación

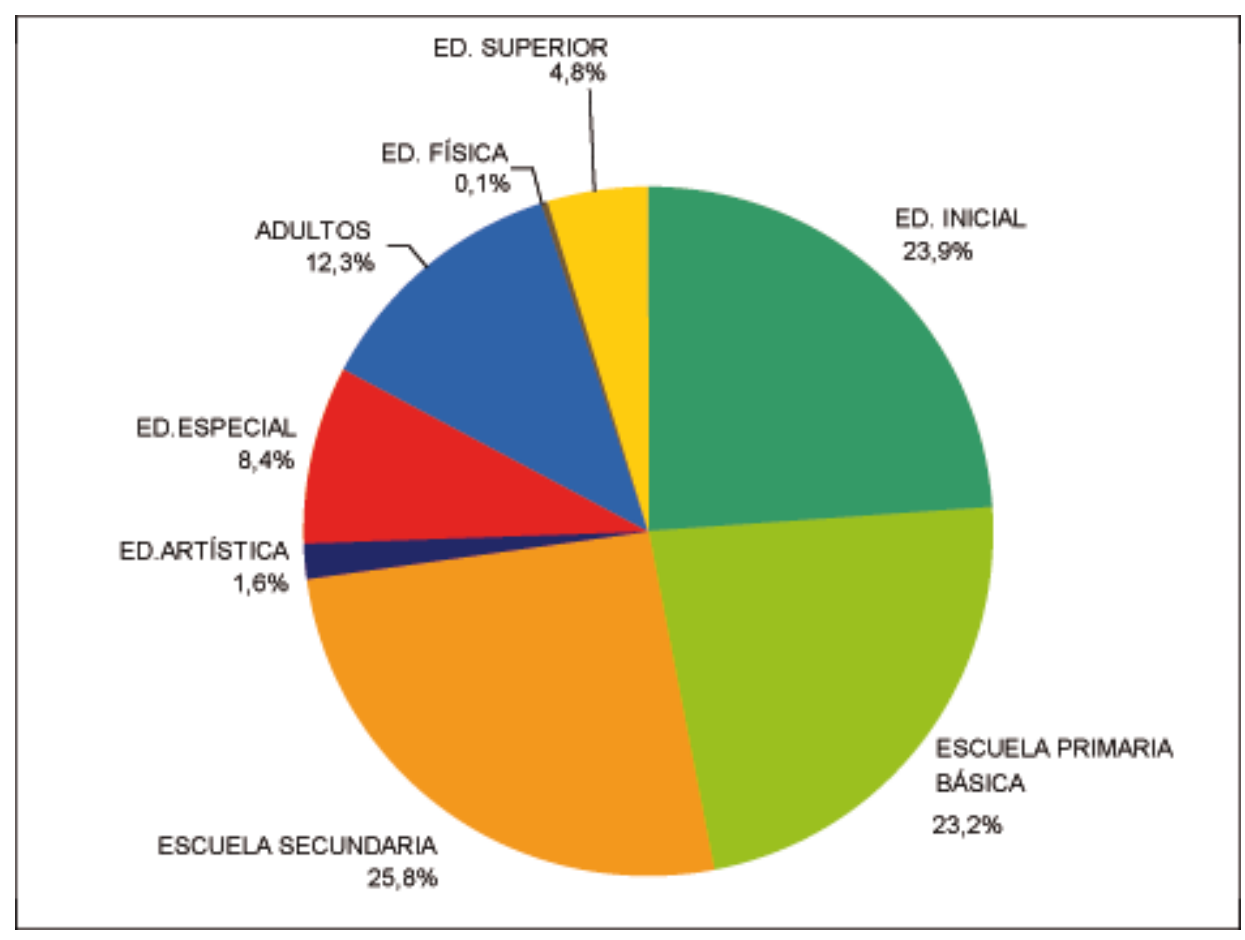

Autor: Elaboración propia

Fuente: http://www.estadistica.laplata.gov.ar/paginas/matricula.htm

Tabla 2: datos de educación de los países 


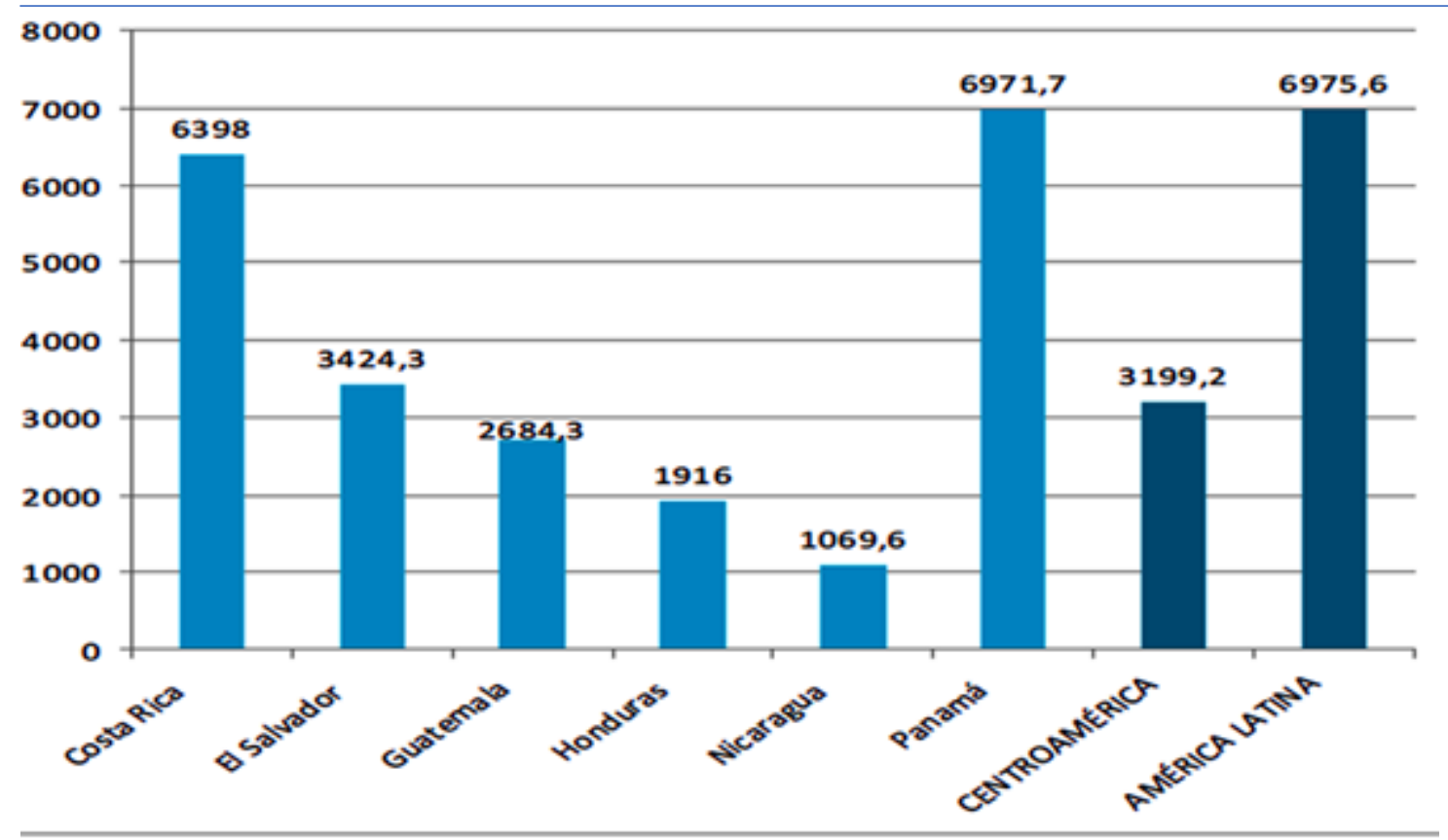

Autor: Carlos López

Fuente: https://www.monografias.com/trabajos98/estudio-comparativosistemas-educativos-centroamerica/estudio-comparativo-sistemas-educativoscentroamerica.shtml

Tabla 3: porcentajes de educación

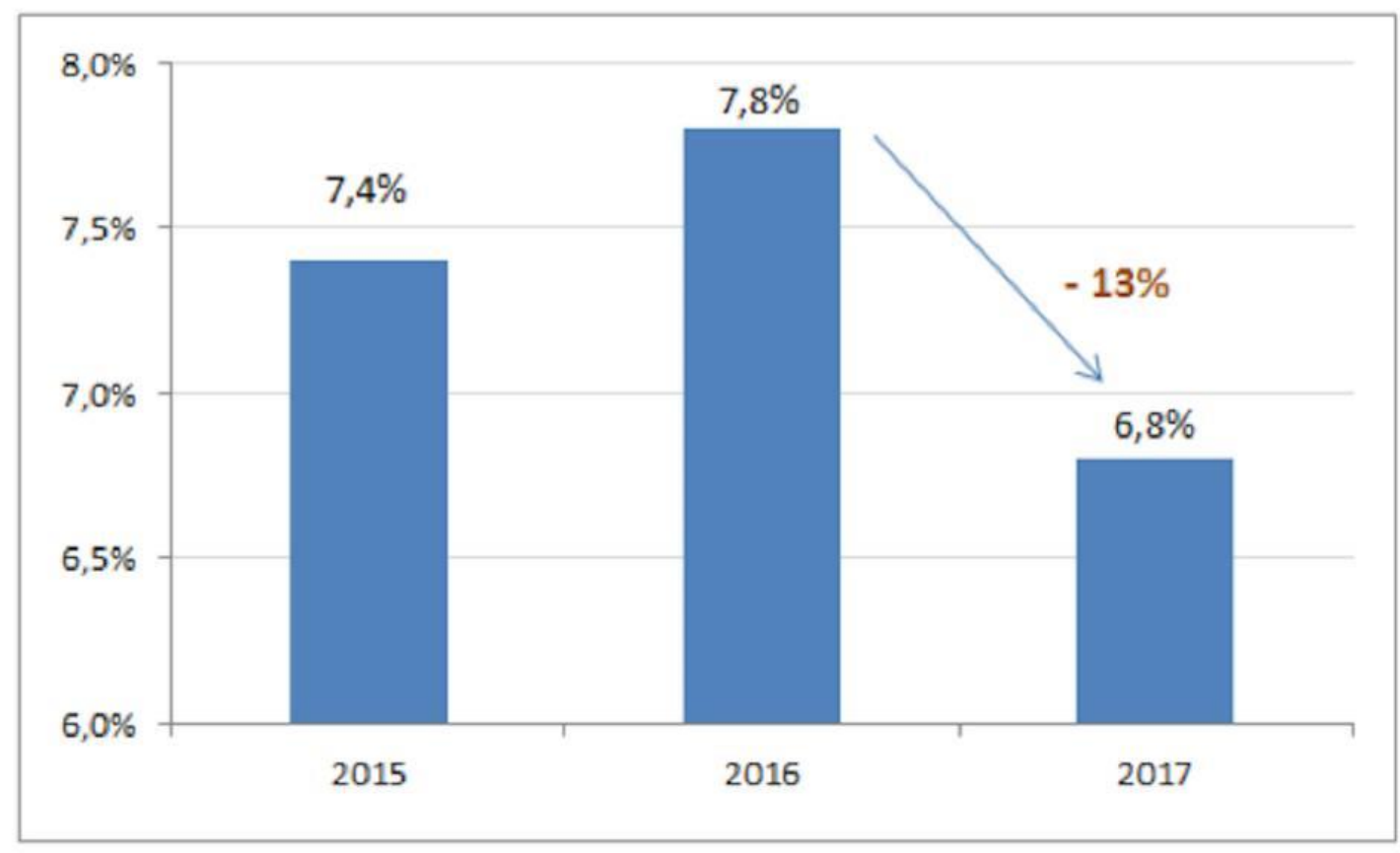

Autor: Aurosa Santiago

Fuente:

https://elpais.com/elpais/2017/09/27/contrapuntos/1506477786_010901.html 
Según Castillo L. 2013., Riuz F.2015 \& Martínez J. 2011 Afirman: Las nuevas tecnología de información y comunicación además del desarrollo del aprendizaje en la educación inicial, tomando en cuenta el avance asombrado de las tecnologías su relación directa entre esta y el surgimiento de estrategias para la operacionalizacion niños/as con gran potencial, en virtud, de las inexistencia de trabas mentales entre el conocimiento. Y las nuevas tecnología se ha logrado un cambio social, los cuales a la vez un conjuntos de innovaciones tecnológicas.

"En la actualidad, la relación computadora- niños constituye un debate que genera aun pocas coincidencia debido a que es un tema nuevo sobre el que falta mucho por aprender es una combinación entre lo teórico-práctico para lograr una integración adecuada de las nuevas tecnologías al nivel inicial."

Las tecnologías de las información y comunicación son una realidad concreta que atraviesa la vida de los sujetos que habitamos las escuelas ya sea forma de teléfonos inteligentes, tabletas, computadoras portátiles, escritorio, entre otros dispositivos circulan y se utiliza a diario.

Pero que sucede cuando eso dispositivos se instalan como objetos de uso u consumo en colegio, escuela y jardines. Las escuelas es una institución que se encuentra inmersa en la sociedad y que no permanece ajena a lo que sucede puertas afuera. En la sociedad del presente, los niños son receptores y productores de información, las nuevas y diversas formas penar en trabajo con la educación inicial es posible si la comprendemos y a las incluimos como un eje transversal en la tarea cotidiana de enseñanzas.

Grafico 6: La educación inicial (TIC)

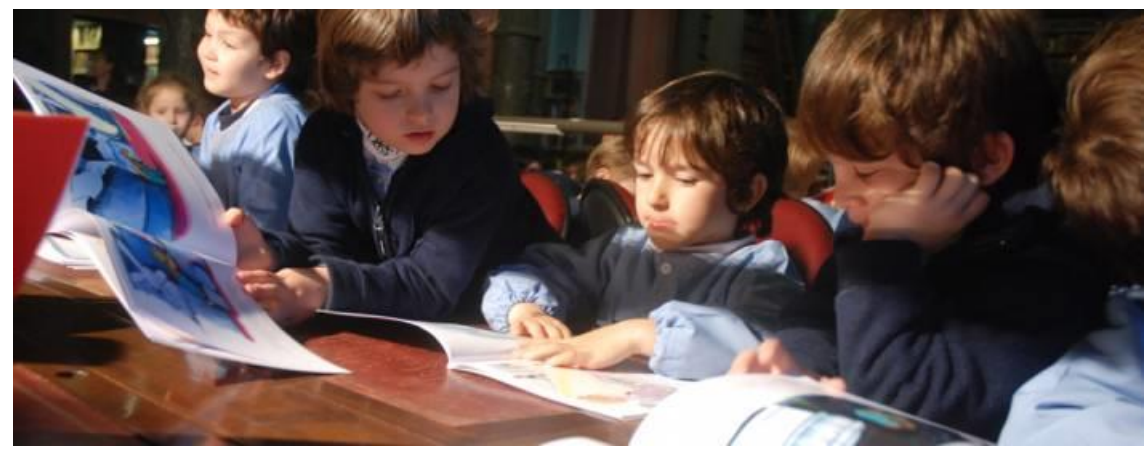

Autor: Educar

Fuente:http://cdn.educ.ar/repositorio/imagen/ver?image_id=d3e377bb-fa5a4a24-a204-a86165ee47b4

El uso de la tecnología educativas como recurso de apoyo para la educación está enriqueciendo el proceso de enseñanzas tradicional, ya que se ha comprobado que mejora el aprendizaje, además de crea condiciones apropiadas para que el estudiante y el profesor, interactúe dentro de un clima practica- aprendizaje. El mundo tecnológico atrae el interés de los niños, para profundizar y ampliar los niveles del conocimiento.

"Las tecnologías en la educación pasan a ser una estrategia de aprendizaje, es decir un conjunto de procedimientos que el estudiante emplea de forma intencional como instrumento flexible para aprender elocuentemente." 
Tabla 4: Datos de educación

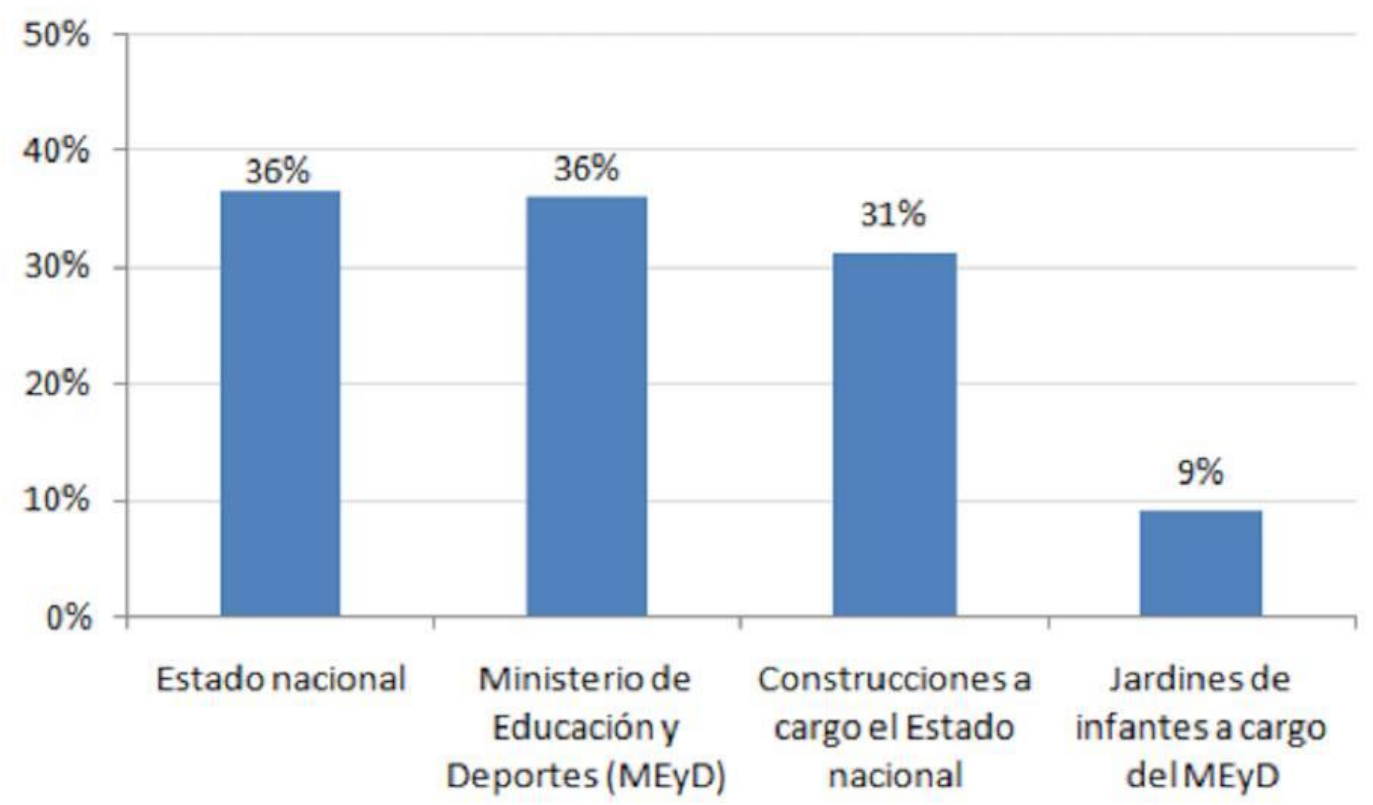

Autor: Pablo Gentili

Fuente:https://elpais.com/elpais/2017/09/27/contrapuntos/1506477786_010901. html

\section{Conclusiones}

> En esta investigación realizada acerca del impacto tecnológico en la educación inicial pude aprecia lo que brindas la tecnología en nuestra vida cotidiana, en el mundo de la enseñanza un papel fundamental que posee de acuerdo al uso que le demos.

$>$ El desarrollo tecnológico ha sido una herramienta para el crecimiento de la sociedad en todos los ámbitos que podamos darle uso a las nuevas tecnologías que viene innovando día a día.

$>$ Debido a la carencia de software apto para los niños y niñas costarricenses, que responda a nuestra realidad, es que la segunda experiencia es relevante, donde las docentes de preescolar participan en la elaboración de software que pueden llegar a usar sus alumnos, de forma tal que se hace valer su criterio y experiencia como especialistas en la educación de niños.

\section{Referencias Bibliográficas}

Barcelo, G. (29 de Diciembre de 2017). Impacto de la Tecnologia en la Sociedad. Tendencia. Recuperado el 14 de Octubre de 2018, de https://www.tendencias21.net/fisica/IMPACTO-DE-LA-CIENCIA-Y-LATECNOLOGIA-EN-LAS-SOCIEDADES_a15.html 
Castillo, L. (2 de Junio de 2013). Tecnologia aplicada. Recuperado el 6 de Noviembre de 2018, de Importacoa de la tecnologia en la educacion incial: http://tecnologiasaplicadaslaurimar.blogspot.com/

Desevilla, A. (19 de Mayo de 2017). Los avances tecnológicos revolucionan la educación. Recuperado el 21 de Octubre de 2018, de Abc: https://sevilla.abc.es/conocer/seviavances-tecnologicos-revolucionan-educacion-201705191138_noticia.html

Fernandez, R. (29 de Abril de 2015). Prezi.com. Recuperado el 14 de Octubre de 2018, de Impacto Tecnologico en el mundo actual: https://prezi.com/pd1ds1dmlitx/elimpacto-de-la-tecnologia-en-el-mundo-actual/

Garcia, M. (27 de Abril de 2013). Tecnologia Educativa en Preescolar. Recuperado el 21 de Octubre de 2018, de preescolarwendymarymonse: http://preescolarwendymarymonse.blogspot.com/

Huidribo, J. M. (15 de Abril de 2018). Los avances tecnologicos y sus impacto e la sociedad. 2cna movilidad. Recuperado el 12 de Octubre de 2018, de https://www.zonamovilidad.es/noticia/18829/los-avances-tecnologicos-y-suimpacto-en-la-sociedad-lo-bueno-y-lo-malo.html

Jarvis, E. (14 de Junio de 2017). BA. Recuperado el 14 de Octubre de 2018, de Impacto de la Tecnologia en el mundo: http://www.buenosaires.gob.ar/noticias/elimpacto-de-la-tecnologia-en-el-mundo-del-trabajo

Martinez, J. (2011). Nuevas tecnologia en la educacion inicial. Educacion Pucp, 20(39). Recuperado el 6 de Noviembre de 2018, de http://revistas.pucp.edu.pe/index.php/educacion/article/view/2488

Martinez, J. (12 de Enero de 2016). Tecnología en el aprendizaje de los niños. Recuperado el 21 de Octubre de 2018, de eligeeducar: https://www.eligeeducar.cl/comoafecta-la-tecnologia-al-aprendizaje-de-los-ninos

Muntz, A. (15 de Febrero de 2017). Randstad. Recuperado el 14 de Octubre de 2018, de Impacto de la Tecnologia: https://www.randstad.es/tendencias360/el-impacto-dela-tecnologia-es-mayor-de-lo-que-imaginas/

Paker, C. (2015). LInked in. Recuperado el 13 de Octubre de 2018, de www.linkedin.com: https://www.linkedin.com/pulse/advancement-newtechnology-positive-negative-colette-parker

Reyes, M. (25 de Abril de 2016). Tecnología y creatividad en niños. Recuperado el 21 de Octubre de 2018, de palabramaestra: https://compartirpalabramaestra.org/alianzagimnasio-campestre-compartir/tecnologia-y-creatividad-en-ninos-de-preescolar 
Riuz, F. (18 de Diciembre de 2015). Educar. Recuperado el 6 de Noviembre de 2018, de las TIC en la educacion incial: https://www.educ.ar/noticias/129250/las-tic-en-laeducacion-inicial

Rodriguez, I. (14 de Julio de 2017). impacto de la tecnologia en los niños. Recuperado el 21 de Octubre de 2018, de La nacion: https://www.nacion.com/ciencia/salud/usode-tecnologia-en-primera-infancia-si-cambia-desarrollo-del-cerebro-pero-nonecesariamente-para-mal/YE6XNYH435H2BJET7ZRTVPNTCE/story/

Rosa, A. (2016). Avances de la tecnologia en la informacion. Mexico: Alfa-Omega. Recuperado el 14 de Octubre de 2018, de http://www.aniei.org.mx/Archivos/Memorias/L_Electronico_CNCIIC2016.pdf

Rowan, C. (19 de Diciembre de 2018). Tecnología en el desarrollo del niño. Recuperado el 21 de Octubre de 2018, de huffingtonpost: https://www.huffingtonpost.es/crisrowan/influencia-de-la-tecnologia-ninos_b_4043967.html

Sigdel, S. (2017). Impact of Technology in Early Childhood. Recuperado el 21 de Octubre de 2018, de scholarsarchive: https://scholarsarchive.jwu.edu/cgi/viewcontent.cgi?referer=https://www.google. com.ec/\&httpsredir $=1 \&$ article $=1055 \&$ context=mba_student

Tabares, J. Q. (26 de Mayo de 2014). Estudio Sociales de la Tecnologia. Iberoamericana de Ciencia, Tecnologia y Sociedad CTS, 9. Recuperado el 13 de Octubre de 2018, de http://www.redalyc.org/pdf/924/92430866007.pdf

\section{【 Ciencia}




\section{Para citar el artículo indexado.}

Velasteguí E. (2017). El avance tecnológico y su impacto en la educación inicial. Revista electrónica Explorador Digital 1(1), 5-17. Recuperado desde:

http://cienciadigital.org/revistacienciadigital2/index.php/exploradordigital/article/view/314/7 26

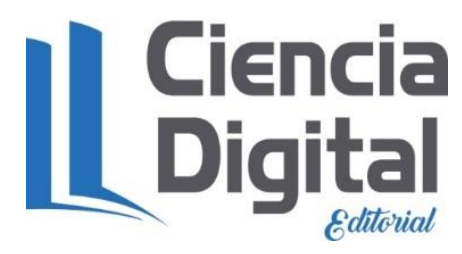

El artículo que se publica es de exclusiva responsabilidad de los autores y no necesariamente reflejan el pensamiento de la Revista Explorador Digital.

El articulo queda en propiedad de la revista y, por tanto, su publicación parcial y/o total en otro medio tiene que ser autorizado por el director o editor de la Revista Explorador Digital.
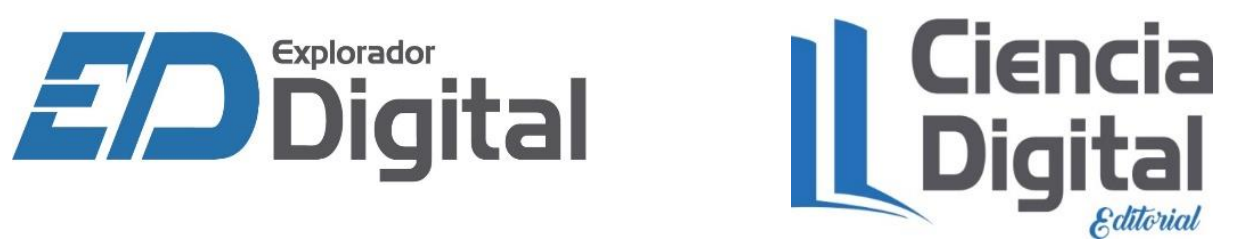